\title{
Relativistic many-body investigation of hyperfine interactions in excited $S$ states of alkali metals: Francium and potassium
}

\author{
Alfred Owusu, R. W. Dougherty, G. Gowri, and T. P. Das \\ Department of Physics, State University of New York at Albany, Albany, New York, 12222 \\ J. Andriessen \\ Technische Natuurkunde, Technische Universiteit Delft, 2628 CJ Delft, The Netherlands
}

(Received 15 April 1996; revised manuscript received 27 January 1997)

\begin{abstract}
To enhance the current understanding of mechanisms contributing to magnetic hyperfine interactions in excited states of atomic systems, in particular, alkali-metal atom systems, the hyperfine fields in the excited $5^{2} S_{1 / 2}-8{ }^{2} S_{1 / 2}$ states of potassium and $8{ }^{2} S_{1 / 2}-12{ }^{2} S_{1 / 2}$ states of francium atoms have been studied using the relativistic linked-cluster many-body perturbation procedure. The net theoretical values of the hyperfine fields for the excited states studied are in excellent agreement with available experimental data for both atoms. There is a significant decrease in importance of the correlation contribution in going from the ground state to the excited states, the correlation contributions as ratios of the direct contribution decreasing rapidly as one moves to the higher excited states. However, the contribution from the exchange core polarization (ECP) effect is nearly a constant fraction of the direct effect for all the excited states considered. Physical explanations are offered for the observed trends in the contributions from the different mechanisms. A comparison is made of the different contributing effects to the hyperfine fields in potassium and francium to those in the related system, rubidium, studied earlier. Extrapolating from our results to the highly excited states of alkali-metal atoms, referred to as the Rydberg states, it is concluded that in addition to the direct contribution from the excited valence electron to the hyperfine fields, a significant contribution is expected from the ECP effect arising from the influence of exchange interactions between electrons in the valence and core states. [S1050-2947(97)07907-9]
\end{abstract}

PACS number(s): 31.10.+z, 31.25.Jf, 31.30.Gs, 31.30.Jv

\section{INTRODUCTION}

First-principle investigations [1,2] of the contributions to the hyperfine interactions in the ground states of alkali-metal atoms by the linked-cluster many-body perturbation theory procedure have provided accurate quantitative explanations of the experimental data in these systems. They have also provided an understanding of the trends followed by the contributions from different physical mechanisms contributing to the hyperfine field in going from the lightest to heaviest alkali-metal atoms. On the other hand, there have not been many first-principle investigations carried out for hyperfine interactions in excited states.

The development of very useful experimental techniques [3-6] for their study has made the understanding of the hyperfine properties of excited states of alkali-metal atoms assume major importance as accurate data have become available in the excited states $[3,7,8]$. With the availability of the experimental data, it is useful to carry out calculations in the excited states not only to explain theoretically these experimental values, but also to examine the trends in contributions from different physical mechanisms in going over the different excited states of the alkali-metal atoms. Such studies are expected to provide, in some respects, more physically interpretable trends than that observed in going over the ground states of different alkali-metal atoms [1,2] since the core states now belonging to the same atom can be considered to vary relatively less in going from one system to the next, which in the present case refer to atomic states with a valence electron in different excited states. In the present work we have carried out, using the relativistic linked-cluster many-body perturbation theory (RLCMBPT) [9], systematic studies of hyperfine interactions on the excited states of potassium and francium for which accurate experimental data are currently available for a number of excited states $[3,7,8,10]$.

In addition to attempting to attain a better understanding of the contributions from different mechanisms to hyperfine properties and their trends over different excited states, there were a number of other motivations for the present study on excited states of potassium and francium. One was to study how the various contributions varied as one went to the highly excited states, referred to in the literature as Rydberg states. In particular, one would like to see if the other mechanisms such as exchange core polarization and many-body effects become negligible in importance and the direct contribution resembles that due to a hydrogen atom. Second, in recent work [11], it has been found that for $\mathrm{Ra}^{+}$ions, manybody contributions to the hyperfine constants for excited states had a negative sign. This is remarkable because in all studies of ground states of alkali-metal atoms and alkaliearth ions the correlation contribution to the hyperfine interaction has always been found to be positive. In trying to explain the origin of the negative correlation contribution for $\mathrm{Ra}^{+}$, it was felt that it would be helpful to study this contribution for the excited states of the isoelectronic neutral francium atom as well as potassium to examine if the corresponding signs for the neutral atoms are always positive, as has been found earlier for rubidium [6]. A third motivation for studying the hyperfine interactions for excited states of 
the alkali-metal atoms is that one can infer from them the expected importance of the various contributions to the hyperfine fields related to Knight shifts [12] in nuclear magnetic resonance measurements in the corresponding alkali metals, which are difficult to obtain quantitatively in the metallic systems.

In Sec. II the basic aspects of the relativistic many-body perturbation procedure $[1,2,9]$ that we have used are given. Some of the details pertinent to the application of the procedure to the excited states are briefly discussed. The important diagrams describing the physical mechanisms contributing to the hyperfine interaction are also presented in this section. In Sec. III the contributions from the important diagrams in our RLCMBPT investigation of the hyperfine fields for the $5 s-8 s^{2} S_{1 / 2}$ states of potassium (K) and $8 s-12 s^{2} S_{1 / 2}$ states of francium $(\mathrm{Fr})$ are presented. These results are discussed with respect to both a comparison with corresponding results in the excited states of $\mathrm{Rb}$ obtained in earlier investigations [13] and the nature of the agreement between the net calculated hyperfine constants obtained by combining the contributions from the various mechanisms and the experimental values for the excited states of $\mathrm{K}[3,8,10]$ and $\mathrm{Fr}[7]$. We shall also discuss in this section the relative importance of the contributions from different mechanisms and the trends followed by them over the different excited states. The main conclusions from our work are given in Sec. IV and the expected natures of the various mechanisms contributing to the highly excited Rydberg states are discussed.

\section{PROCEDURE}

The RLCMBPT procedure employed in our present work on excited states of potassium and francium has been extensively used in the literature to calculate various properties of a number of atomic systems. The major aspects of the theory have been described in detail in earlier papers [1,2,9]. We shall therefore give only a brief summary of the theory for the sake of completeness and to provide the background for some points in the discussion of our results in Sec. III.

The RLCMBPT procedure essentially evaluates the expectation value of the hyperfine operator over the exact relativistic eigenfunction of the atomic many-particle Hamiltonian obtained [9] using the linked-cluster expansion in perturbation theory starting with the relativistic Hartree-Fock Hamiltonian as zeroth order. The occupied states including the valence and core states are generated by the relativistic Hartree-Fock procedure [14] in which the potential employed is referred to as the $V^{N}$ potential [9]. The excited states needed for application of perturbation theory are generated using the $V^{N-1}$ potential $[1,2,9]$ based on the occupied core states obtained by the relativistic Hartree-Fock procedure and omitting the self-Coulomb and self-exchange potential associated with the valence electron. The $V^{N-1}$ potential is used because it is physically more meaningful $[1,9,13]$ for the excited states, providing a potential that looks, at large distances for neutral atoms, as if it arises effectively from a single positive charge at the nucleus in contrast to essentially a zero potential for the $V^{N}$ potential. The result is that the spectrum of excited states is richer for the $V^{N-1}$ potential involving bound as well as continuum excited states, in contrast to only continuum states for the

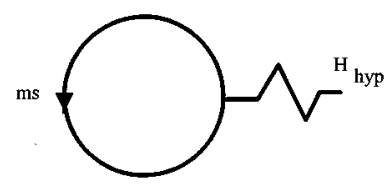

(a)

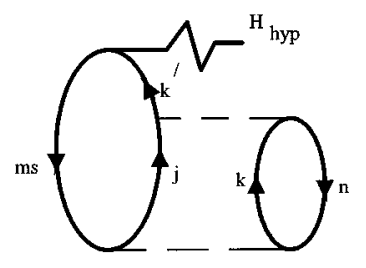

(c)

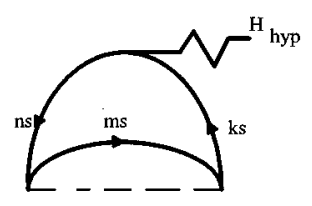

(b)

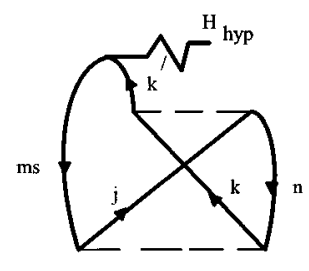

(d)
FIG. 1. Zero-, first-, and second-order diagrams contributing the direct, exchange core polarization, and correlation contributions to the hyperfine interactions in potassium and francium excited states.

$V^{N}$ potential, leading to much faster convergence $[1,9,13]$ in perturbation theory when the $V^{N-1}$ potential is used. For the particle (unoccupied) states of particular $l$ we generate bound states with principal quantum number $n$ up to and including $n=12$ and 15 continuum states with energies corresponding to the mesh points of a 15-point Gauss-Laguerre quadrature formula [15]. Since in earlier work $[16,17]$ the Coulomb potential associated with the volume distribution of the nuclear charge has been shown to have a significant influence on the hyperfine interaction especially for the heavy atoms, we have used a model of a uniform charged sphere for the nucleus, the total nuclear charge $\zeta e$ being uniformly distributed throughout the volume of a sphere with a radius of $1.2 A^{1 / 3} \mathrm{fm}, A$ being the mass number of the nucleus.

The individual terms in the expansion are expressed as diagrams [1] and can be associated with specific physical effects $[1,2,9]$. The algebraic expressions associated with various diagrams, used in their evaluation, have been discussed in the literature $[1,2,9,13]$. Typical diagrams that represent the various physical effects (the direct, exchange core polarization, and correlation terms) are shown in Fig. 1. Figure 1(a) is the diagrammatic representation of the direct or zeroth-order Hartree-Fock contribution, arising from the unpaired spin valence electron. The exchange core polarization (ECP) effect is represented in Fig. 1(b). This effect represents the influence of the preferential exchange interaction between the valence electron and the core electrons with spin parallel to the former, leading to a net spin density at the nucleus due to the core electrons. Any diagram in which two electrons are simultaneously excited belongs to the family of many-body or correlation diagrams. Thus the major correlation diagram in Fig. 1(c) represents simultaneous excitation of the valence $m s$ electron and a core $n$ electron to unoccupied states $j$ and $k$ through their mutual interaction, a second such interaction leaving only the valence electron excited and contributing to the hyperfine constant. Figure $1(d)$ is the exchange counterpart of Fig. 1(c), the excitation of electrons in states $m s$ and $n$ being followed by an exchange of roles between the excited states $j$ and $k$.

We would like to make some remarks about the one- 
TABLE I. Contributions to the hyperfine field (in tesla) at ${ }^{39} \mathrm{~K}$ from excited states in potassium from different mechanisms.

\begin{tabular}{lcccccrc}
\hline \hline State & Direct & ECP & Corr. & $\begin{array}{c}\text { ECP } \\
\text { Direct }\end{array}$ & $\begin{array}{c}\text { Corr. } \\
\text { Direct }\end{array}$ & Total & Expt. $^{{ }^{a}}$ \\
\hline $4^{2} S_{1 / 2}$ & 37.60 & 7.73 & 11.38 & 0.24 & 0.30 & 56.71 & 58.02 \\
$5^{2} S_{1 / 2}$ & 9.96 & 2.35 & 1.71 & 0.24 & 0.17 & 14.02 & 13.95 \\
$6^{2} S_{1 / 2}$ & 3.78 & 1.12 & 0.62 & 0.29 & 0.16 & 5.52 & 5.48 \\
$7^{2} S_{1 / 2}$ & 2.12 & 0.54 & 0.26 & 0.25 & 0.12 & 2.92 & 2.71 \\
$8^{2} S_{1 / 2}$ & 1.16 & 0.28 & 0.14 & 0.24 & 0.12 & 1.58 & 1.50 \\
\hline \hline
\end{tabular}

${ }^{\mathrm{a}}$ References [3, 8, 10].

electron basis functions used in the evaluation of the diagrams before presenting our results for the excited states of potassium and francium. The unperturbed determinant for the atomic ground state $4{ }^{2} S_{1 / 2}$ of potassium, for example, is built out of the one-electron wave function $\varphi_{n}$ corresponding to the core states and the $4 s$ valence state, all $m s$ states with $m>4$ being considered excited orbitals. The excited $5{ }^{2} S_{1 / 2}$ state, however, is represented by a determinant $\Phi_{0}$ made up of the same core state orbitals, but with $5 s$, instead of $4 s$, being the valence state function. Consequently, in addition to the $m s>5 s$ states corresponding to the excited states, the $4 s$ state is also considered as an excited $s$ state in carrying out the evaluation of the perturbation diagrams. The latter feature is a consequence of the fact that even though the one-electron state $4 s$ has an energy lower than the $5 s$ state in the $5{ }^{2} S_{1 / 2}$ multiplet state, it is empty and thus available as an "excited" or particle state in the diagrams of many-body theory. Similarly, for the $6{ }^{2} S_{1 / 2}, 7{ }^{2} S_{1 / 2}$, and $8^{2} S_{1 / 2}$ states, the set of excited $s$ states are, respectively, $(4 s, 5 s, 7 s, 8 s, \ldots),(4 s, 5 s, 6 s, 8 s, 9 s, \ldots)$, and $(4 s, 5 s, 6 s, 7 s, 9 s, 10 s, \ldots)$. The higher-angular-momentum states $(4 p, 5 p, 6 p, \ldots),(3 d, 4 d, 5 d, \ldots),(4 f, 5 f, 6 f, \ldots)$, and $(5 g, 6 g, 7 g, \ldots)$ in the $V^{N-1}$ potential chosen are the oneelectron excited $p, d, f$, and $g$, states, respectively, for the multiplet states $m^{2} S_{1 / 2}(m=5,6,7$, and 8), just as in the case of the ground state $4{ }^{2} S_{1 / 2}$. Correspondingly, for francium, the unperturbed many-electron excited $8{ }^{2} S_{1 / 2}$ state is represented by a determinant that is made up of the same core state orbitals as in the case of the many-electron ground $7^{2} S_{1 / 2}$ state, but with the $8 s$ as the valence state. The $7 s$ as well as $m s>8 s$ states are now considered as excited states for perturbation calculations for the $8{ }^{2} S_{1 / 2}$ state. Similar to the case of potassium, in the Fr atom, the $7 s$ state is lower in energy than the $8 s$ state in the $8{ }^{2} S_{1 / 2}$ multiplet state; however, it is empty and therefore available as an "excited state." In the same way for the $9^{2} S_{1 / 2}, 10^{2} S_{1 / 2}$, $11^{2} S_{1 / 2}$, and $12{ }^{2} S_{1 / 2}$ multiplet states, the set of excited $s$ states is $(7 s, 8 s, 10 s, 11 s, \ldots), \quad(7 s, 8 s, 9 s, 11 s, 12 s, \ldots)$, $(7 s, 8 s, 9 s, 10 s, 12 s, \ldots)$, and $(7 s, 8 s, 9 s, 10 s, 11 s, 13 s, \ldots)$, respectively, the higher-angular-momentum excited states being the same as for the many-electron ground $7{ }^{2} S_{1 / 2}$ state. In addition to the fact that it is important to use for the excited states in perturbation diagrams the unoccupied basis states in the $V^{N-1}$ potential, there are some questions of detail as to which occupied states should be used to obtain the $V^{N-1}$ potential because we are dealing with the hyperfine property for excited many-electron states. Thus, considering the $8{ }^{2} S_{1 / 2}$ state, the most accurate procedure would be to gen- erate all the occupied states in the Hartree-Fock approximation with the valence electron in the excited $8 s$ state and then use this valence electron state wave function and the core wave functions to create the $V^{N-1}$ potential in which to generate the unoccupied states. This would, however, be unduly time consuming when one wants to study a number of many electron excited states. In this connection, in our earlier work involving many-electron excited states of $\mathrm{Ra}^{+}$ion [11], the time-saving approximation was made of using the same core wave functions as for the ground $7{ }^{2} S_{1 / 2}$ configuration and the corresponding $V^{N-1}$ potential was used to determine the excited states, employing the $8 s$ state in this potential as the occupied valence state. The calculated hyperfine constant with this approach differed by less than $1 \%$ compared to that obtained using the occupied and unoccupied states determined for the $8{ }^{2} S_{1 / 2}$ configuration. We have therefore used this time-saving procedure for all the excited configurations studied for potassium and francium.

Before concluding this section we would like to make one more remark about the procedure used. The evaluation of the diagrams in RLCMBPT requires a complete set of basis functions not only in terms of the principal quantum numbers $n$ for the bound excited states and the number of continuum states, but also the angular-momentum quantum numbers $l$ for the bound and continuum excited states. The nature of the convergence with respect to $l$ observed in earlier work [9] in ground states of alkali-metal atoms indicated that it was sufficient to use up to $g$ states in evaluating the diagrams, which is followed here.

\section{RESULTS AND DISCUSSION}

The main results of our investigation of the hyperfine fields for the excited states of potassium and francium will now be presented and discussed. The various contributions to the hyperfine fields $H_{\text {direct }}, H_{\mathrm{ECP}}$, and $H_{\text {corr }}$ at the nucleus for Fr and $\mathrm{K}$ from our investigation are listed in Tables I and Tables II, respectively. Also listed for ready reference are the experimental results for the hyperfine fields in the states where they are available. We will first compare our theoretically obtained results with experiment and then proceed to analyze the trends observed in the variation of the contributions from direct, ECP, and correlation effects in going from the ground to the highest multiplet state $m^{2} S_{1 / 2}$ studied. We shall also compare these contributions, especially as fractions of direct contributions, with the results for $\mathrm{Rb}$ excited states (Table III) that have been obtained earlier by the present theory. The first rows in Tables I-III represent the 
TABLE II. Contributions to the hyperfine fields (in tesla) at ${ }^{212} \mathrm{Fr}$ from excited states in francium from different mechanisms.

\begin{tabular}{lrrrrrrr}
\hline \hline State & Direct & ECP & Corr. & $\begin{array}{c}\text { ECP } \\
\text { Direct }\end{array}$ & $\begin{array}{c}\text { Corr. } \\
\text { Direct }\end{array}$ & Total & Expt. $^{\text {a }}$ \\
\hline $7^{2} S_{1 / 2}$ & 456.10 & 75.30 & 123.80 & 0.17 & 0.27 & 655.20 & \\
$8^{2} S_{1 / 2}$ & 126.84 & 18.84 & 21.78 & 0.15 & 0.17 & 167.46 & \\
$9^{2} S_{1 / 2}$ & 50.74 & 7.54 & 6.94 & 0.15 & 0.14 & 65.22 & \\
$10 S_{1 / 2}$ & 24.83 & 3.82 & 2.66 & 0.15 & 0.11 & 31.31 & 33.72 \\
$11 S_{1 / 2}$ & 13.16 & 2.10 & 0.91 & 0.16 & 0.07 & 16.17 & 18.92 \\
$12 S_{1 / 2}$ & 7.24 & 1.13 & 0.31 & 0.16 & 0.04 & 8.68 & \\
\hline \hline
\end{tabular}

${ }^{\mathrm{a}}$ References [6, 7].

results for the ground states, which are listed here for the sake of comparison with the excited multiplet $m^{2} S_{1 / 2}$ states.

From Tables I and II it can be seen that for both potassium and francium the largest contribution comes from the direct effect for both the ground states and the excited states. This contribution ranges from $66 \%$ of the experimental value in the ground state to $77 \%$ in the highest excited state $\left(8{ }^{2} S_{1 / 2}\right)$ considered for $\mathrm{K}$ and $69.6 \%$ of experimental value in the ground state to $83.4 \%$ in the highest excited state $\left(12{ }^{2} S_{1 / 2}\right)$ in Fr. The third columns in both tables give the ECP contribution for each system. The correlation contributions are shown in the fourth columns of both tables and the net sums of contributions from all the physical mechanisms in the seventh columns. The fifth and sixth columns present the ratios of the ECP and correlation contributions to the direct contribution and the experimental data for the net hyperfine constants are given in the eighth column. A comparison of our net calculated hyperfine fields with the available experimental data from Tables I and II shows very good agreement between theory and experiment, in the range 9698\% for the excited states of potassium and $95-98 \%$ for francium. This good agreement obtained between the theoretical and experimental hyperfine fields provides confidence in the accuracy of the contributions from individual mechanisms.

We shall now discuss the trends observed in the contributions from different mechanisms in going from the ground state to the excited states. From our results in Table I for K, the direct contribution decreases continuously as one moves from the ground state to the higher excited states, the same trend being also observed for Fr (Table II). This is of course expected since the valence orbital gets progressively less bound as one goes to higher excited states. However, although there is this decrease as one goes to the excited states, the direct contributions progressively represent an increasing fraction of the total hyperfine field, as a result of the combination of ECP and correlation effects becoming decreasing fractions of the total hyperfine field as seen from Tables I and II. A principal reason for this trend is that the two mechanisms of ECP and correlation contributions represent effects produced by the interactions between the valence and the core electrons. As we go to successively higher excited states, the valence electron becomes more loosely bound and its effective separation from the core electrons increases. The interaction between the core and valence electrons therefore decreases and this results in a decrease or weaker perturbation associated with the ECP and correlation effects.

It can also be seen from Tables I and II that the ratio $H_{\mathrm{ECP}} / H_{\text {direct }}$ as a function of the principal quantum numbers of the excited states is nearly a constant for both atoms. The cause for this near constancy of $H_{\mathrm{ECP}} / H_{\text {direct }}$ is that both the direct density at the nucleus due to the valence electron responsible for $H_{\text {direct }}$ and its overlap with the core states related to the valence-core exchange interaction leading to $H_{\mathrm{ECP}}$ decrease with higher excitations of the valence state.

The correlation contribution $H_{\text {corr }}$ is seen from Tables I and II to fall rapidly in value as one goes to the higher excited states. More meaningful are the ratios of the manybody contributions to the direct results, namely, $H_{\text {corr }} / H_{\text {direct }}$. This ratio, unlike that of ECP, undergoes a rapid decrease for both $\mathrm{K}$ and $\mathrm{Fr}$ in going to higher excited states, the ratio for the ground $4{ }^{2} S_{1 / 2}$ state of $\mathrm{K}$ being $30 \%$, while for the highest excited state studied $\left(8^{2} S_{1 / 2}\right)$ it is only $12 \%$. In $\mathrm{Fr}, H_{\text {corr }} / H_{\text {direct }}$ decreases from $27 \%$ in the ground state $\left(7^{2} S_{1 / 2}\right)$ to as low as $4 \%$ in the highest excited state $\left(12^{2} S_{1 / 2}\right)$ studied. The explanation of this decrease for higher excited valence states can be considered as arising

TABLE III. Contributions to the hyperfine field (in tesla) at ${ }^{87} \mathrm{Rb}$ from excited states in rubidium from different mechanisms.

\begin{tabular}{lrrrrrrr}
\hline \hline State & Direct $^{\mathrm{a}}$ & ECP $^{\mathrm{a}}$ & Corr. $^{\mathrm{a}}$ & $\begin{array}{c}\text { ECP } \\
\text { Direct }\end{array}$ & $\begin{array}{c}\text { Corr. } \\
\text { Direct }\end{array}$ & Total $^{\mathrm{a}}$ & Expt. $^{\text {b }}$ \\
\hline $5^{2} S_{1 / 2}$ & 80.6 & 13.9 & 29.6 & 0.17 & 0.36 & 123.4 & 122.2 \\
$6^{2} S_{1 / 2}$ & 21.2 & 3.4 & 4.7 & 0.16 & 0.22 & 29.3 & 28.9 \\
$7{ }^{2} S_{1 / 2}$ & 8.7 & 1.4 & 1.4 & 0.16 & 0.16 & 11.5 & 11.4 \\
$8^{2} S_{1 / 2}$ & 4.4 & 0.7 & 0.5 & 0.16 & 0.11 & 5.6 & 5.7 \\
\hline \hline
\end{tabular}

${ }^{\text {a Reference [13]. }}$

${ }^{\mathrm{b}}$ Reference [6]. 
from the weakening of the van der Waals type of pair correlation interaction $[1,2,9]$ between the core and valence electrons due to the larger effective separation between the two for the excited valence states. The higher excited states are of course more deformable than the lower ones and the ground state and this would tend to enhance the correlation contribution. The observed rapid decrease in $H_{\text {corr }} / H_{\text {direct }}$ in Tables I and II indicates that the effect of the increasing separation between the valence and core electrons as one goes to higher excited states dominates over the influence of the increasing deformability of the valence electron and this determines the direction of the trend in correlation effects. These features obtained in both $\mathrm{K}$ and $\mathrm{Fr}$ are all similar to those observed in Table III from earlier work on $\mathrm{Rb}$ [13] and suggest that the general nature of the conclusions in the present work would apply to all alkali-metal atoms.

\section{CONCLUSION}

The investigations in this paper on the excited states of neutral atoms of $\mathrm{K}$ and $\mathrm{Fr}$ and earlier on Rb have shown that for those states where experimental data are available, one obtains very good agreement between experiment and theory. However, this happens only after one includes significant contributions from exchange core polarization and correlation effects in addition to direct contributions, providing support for the accuracy of our results for these latter contributions.

The trends found in the exchange core polarization and correlation contributions to the hyperfine field suggest that even for highly excited states such as the Rydberg states, the core electrons cannot be approximated as essentially negative point charges at the nuclear site, reducing the effective charge experienced by the valence electron to that due to a single positive charge as appears to be the case for the energies of these states. One has instead to consider the detailed interactions of the core electrons with the valence electron at least for the exchange effect leading to the exchange core polarization contribution to the hyperfine field. It would be helpful to have experimental results for the Rydberg states for $\mathrm{K}, \mathrm{Fr}$, and $\mathrm{Rb}$, and indeed all the alkali-metal atoms, to verify this conclusion.
[1] E. S. Chang, R. T. Pu, and T. P. Das, Phys. Rev. 174, 1 (1968); M. Vajed-Samii, J. Andriessen, B. P. Das, S. N. Ray, T. Lee, and T. P. Das, J. Phys. B 15, L379 (1982); S. N. Panigrahy, R. W. Dougherty, J. Andriessen, and T. P. Das, Phys. Rev. A 40, 1765 (1989).

[2] X. Yuan, S. N. Panigrahy, R. W. Dougherty, J. Andriessen, and T. P. Das, Phys. Rev. A 52, 197 (1995), and references therein.

[3] R. Gupta, W. Happer, L. K. Lam, and S. Svanberg, Phys. Rev. A 8, 2792 (1973); P. Tsekeris, J. Farley, and R. Gupta, ibid. 11, 2202 (1975); J. Farley, P. Tsekeris, and R. Gupta, ibid. 15, 1530 (1977).

[4] R. Gupta, S. Chang, and W. Happer, Phys. Rev. A 6, 529 (1972); R. Gupta, S. Chang, C. Tai, and W. Happer, Phys. Rev. Lett. 29, 659 (1972).

[5] K. H. Liao, L. K. Lam, R. Gupta, and W. Happer, Phys. Rev. Lett. 32, 1340 (1974); see also E. Arimondo, M. Inguscio, and Violino, Rev. Mod. Phys. 49, 31 (1977).

[6] S. Liberman, J. Pinard, H. T. Duong, P. Juncar, P. Pillet, J. L. Vialle, P. Jacquinot, F. Touchard, S. Buttgenbach, C. Thibault, M. De Saint-Simon, R. Klapisch, A. Pesnelie, and G. Huber, C. R. Acad. Sci. B 268, 253 (1978).

[7] E. Arnold, W. Borchers, H. T. Duong, P. Juncar, J. Lermé, W. Neu, R. Neugart, M. Pellarin, J. Pinard, J. L. Vialle, K. Wendt, and ISOLDE Collaboration, J. Phys. B 23, 3511 (1990); A. Coc, C. Thibault, F. Touchard, H. T. Duong, P. Juncar, S. Liberman, J. Pinard, J. Lerme, J. L. Vialle, S. Buttgenbach, A. C. Mueller, A. Pesnelle, and ISOLDE Collaboration., Phys. Lett. 163B, 66 (1985).
[8] P. Tsekeris, R. Gupta, W. Happer, G. Belin, and S. Svanberg, Phys. Lett. 48A, 101 (1974).

[9] T. P. Das, Hyperfine Interact. 34, 189 (1987).

[10] G. Belin, L. Holmgren, I. Lindgren, and S. Svanberg, Phys. Scr. 12, 287 (1975).

[11] A. Owusu, X. Yuan, R. W. Dougherty, G. Gowri, J. Andriessen, and T. P. Das, Bull. Am. Phys. Soc. 40, 142 (1995); A. Owusu, Ph.D. thesis, State University of New York at Albany, 1995 (unpublished).

[12] S. D. Mahanti, L. Tterlikkis, and T. P. Das, in Magnetic Resonance, edited by C. K. Coogan, N. S. Ham, S. N. Stuart, J. R. Pilbrows, and G. V. H. Wilson (Plenum, New York, 1970); S. D. Mahanti and T. P. Das, Phys. Rev. B 4, 46 (1971).

[13] M. Vajed-Samii, S. N. Ray, and T. P. Das, Phys. Rev. A 24, 1204 (1981).

[14] I. P. Grant, Proc. R. Soc. London, Ser. A 262, 555 (1961); T. P. Das, Relativistic Quantum Mechanics of Electrons (Harper \& Row, New York, 1973); H. A. Bethe and E. E. Salpeter, Quantum Mechanics of One- and Two-Electron Systems (Springer, Berlin, 1957); J. Andriessen, K. Raghunathan, S. N. Ray, and T. P. Das, Phys. Rev. B 15, 2533 (1977).

[15] A. H. Stroud and Don Secrest, Gaussian Quadrature Formulas (Prentice-Hall, Englewood Cliffs, NJ, 1966).

[16] V. A. Dzuba, V. V. Flambaum, and O. P. Shuskov, J. Phys. B 17, 1953 (1984).

[17] M. Vajed-Samii, J. Andriessen, B. P. Das, S. N. Ray, T. Lee, and T. P. Das, Phys. Rev. Lett. 48, 1330 (1982); 49, 1466 (1982); 49, 1800 (1982). 